\title{
Guaranteed cost fuzzy controllers for a class of uncertain nonlinear dynamic systems
}

\author{
NATACHE S.D. ARRIFANO and VILMA A. OLIVEIRA \\ Departamento de Engenharia Elétrica, Universidade de São Paulo, USP \\ Av. Trabalhador São-carlense, 400 - 13566-550 São Carlos, SP, Brasil \\ E-mails: natachea@sel.eesc.usp.br/vilmao@sel.eesc.usp.br
}

\begin{abstract}
Stability analysis and a systematic robust fuzzy control design for uncertain nonlinear systems with polytopic uncertainties are presented. The approach given uses the T-S fuzzy systems which are represented by a family of local uncertain linear systems with aggregation. The resulting fuzzy controller is a blending of state feedback gains obtained from a guaranteed cost control (GCC) problem. A sufficient condition for the robust stability of the feedback T-S fuzzy system with uncertainties using a quadratic Lyapunov function is given. A feature of the proposed approach is that an upper bound on the guaranteed cost is minimized by solving an optimization problem with linear matrix inequalities (LMIs).
\end{abstract}

Mathematical subject classification: 93C42, 93B51, 34D20.

Key words: guaranteed cost control, fuzzy control, robust control, uncertain T-S fuzzy systems.

\section{Introduction}

The main difficulties found in the control design for real systems are the nonlinearities and uncertainties. If we treat nonlinearities as uncertainties the obtained results for stability are in general conservatives. In the last years, attractive stability results for nonlinear systems using Takagi-Sugeno (T-S) fuzzy systems [1] based on the theory of stability of nonlinear systems appeared in Feng et al. [2] and $\mathrm{Cao}$ et al. [3]. In the same context, stability conditions using LMIs were given in Hong and Langari [4], Li et al. [5] and Wang et al. [6]. Following this trend, the robust stabilization of uncertain nonlinear systems became an active field of research wherein available robust control techniques for uncertain 
linear systems is extended to deal with the T-S fuzzy systems [7]-[10]. In the framework of T-S fuzzy systems, parametric uncertainty can be represented in general by norm-bounded or polytopic uncertain sets. Different robust control solutions for the T-S fuzzy system with norm-bounded and polytopic uncertainty representation can be found in Lee et al. [7] and Tanaka et al. [8] and Cao et al. [9], respectively. In Cao et al. [9] it is developed a constructive algorithm to the $H_{\infty}$ feedback control of uncertain nonlinear systems based on a piecewise differentiable quadratic Lyapunov function. They accomplish this in a rather involved manner by decomposing the T-S fuzzy system into independent subsystems of the state space representing the crisp, boundary and transition regions. The latter subsystem is in turn decomposed into a set of extreme subsystems. The motivation for this approach is the fact that one can associate a Lyapunov function to each subsystem. In this paper, we follow Cao et al. [9] to represent the uncertain nonlinear dynamic systems with polytopic uncertainties but we consider a common Lyapunov function to establish the stability for the global T-S fuzzy system, thus simplifying the solution. In the context of LMIs, we give a computationally tractable solution to the design of guaranteed cost fuzzy controllers (GCFC) and stability analysis of real systems which can be represented by this class of uncertain nonlinear systems.

We consider a class of uncertain nonlinear dynamic systems described by

$$
\dot{x}(t)=f(x(t), p)+g(x(t), p), u(t)
$$

where $x(t) \in R^{n}$ is the system state vector, $u(t) \in R^{m}$ is the input vector, $p \in R^{N}$ is the uncertain parameter vector, $f():. R^{n} \times R^{N} \rightarrow R^{n}$ and $g():. R^{n} \times R^{N} \rightarrow$ $R^{n \times m}$ are uncertain nonlinear functions. We assume a polytopic representation of (1) as

$$
\dot{x}(t)=\sum_{k=1}^{\nu} \eta_{k}(p)\left(f_{k}(x(t))+g_{k}(x(t)) u(t)\right)
$$

for

$$
k=1,2, \ldots, v, \eta_{k}(p) \geq 0, \sum_{k=1}^{\nu} \eta_{k}(p)=1,
$$


where $f_{k}($.$) and g_{k}(),. k=1,2, \ldots, v$ define the called vertex systems, with the number of vertexes equal to $v\left(v=2^{N}\right.$; $\mathrm{N}$ the number of uncertain parameters of the system).

Local uncertain linear models of (2) are constructed by extending the linearization formula proposed by Teixeira and Zak [10], which yields a good approximates of the system in the vicinity of the operating point even if the operating point is not an equilibrium point. The T-S fuzzy system adopted in this work is inferred as a weighted average of local input-output relations of (2) and can be represented by

$$
\dot{x}(t)=\sum_{i=1}^{r} \alpha_{i}(x(t))\left(A_{i}(p) x(t)+B_{i}(p) u(t)\right)
$$

where $A_{i}($.$) and B_{i}(),. i=1,2, \ldots, r$ describe the uncertain linear systems linearized in $r$ chosen operation points, $x(t)$ are the premise variables, $\alpha_{i}(),. i=$ $1,2, \ldots, r$ are the normalized membership functions, such that $\alpha_{i}(x(t)) \geq 0$ for $i=1,2, \ldots, r$ and $\sum_{i=1}^{r} \alpha_{i}(x(t))=1[8]$.

The remainder of the paper is organized as follows: Section 2 presents the stability analysis and the GCFC design. Simulation results on a mass-springdamper mechanical system are given in Section 3 to illustrate the effectiveness of the proposed approach. The paper concludes with brief remarks in Section 4.

\section{Fuzzy control design for T-S fuzzy systems with uncertainties}

In the fuzzy control design, we use the concept of parallel distributed compensation (PDC), where each controller is distributively designed for the corresponding rule of the T-S fuzzy system [6]. Therefore, the fuzzy controller for T-S fuzzy system (3) is inferred as a weighted average of the controllers designed for each rule,

$$
u(t)=-\sum_{i=1}^{r} \alpha_{i}(x(t)) F_{i} x(t)
$$

where $F_{i} \in R^{m \times n}, i=1,2, \ldots, r$ are the local state feedback gains to be designed with $\alpha_{i}$ (.), $i=1,2, \ldots, r$ as defined before. Substituting (4) in (3), 
we obtain the state feedback T-S fuzzy system as

$$
\dot{x}(t)=\sum_{i=1}^{r} \sum_{j=1}^{r} \alpha_{i}(x(t))\left(A_{i}(p)-B_{i}(p) F_{i}\right) x(t)
$$

Using (2) and some algebraic manipulations, we can write (5) as

$$
\begin{aligned}
\dot{x}(t)= & \sum_{i=1}^{r} \alpha_{i}^{2}(x(t))\left[\sum_{k=1}^{v} \eta_{k}(p) H_{i k}\right] x(t) \\
& +\sum_{i<j}^{r} \alpha_{i}(x(t)) \alpha_{j}(x(t))\left[\sum_{k=1}^{v} \eta_{k}(p) G_{i j k}\right] x(t)
\end{aligned}
$$

where $H_{i k}:=A_{i k}-B_{i k} F_{i}$ and $G_{i j k}:=A_{i k}-B_{i k} F_{j}+A_{j k}-B_{j k} F_{i}$ for $i=1,2, \ldots, r$ and $k=1, \ldots, v$. In (6), notation $\sum_{i<j}^{r}$ means, for instance for $r=3, \sum_{i<j}^{3} \alpha_{i j} \Leftrightarrow \alpha_{12}+\alpha_{13}+\alpha_{23}$.

\subsection{Stability analysis}

In this section, we derive a sufficient condition for the stability of the feedback T-S fuzzy system (6) in terms of a Lyapunov function candidate of the type $V(x(t))=x(t)^{T} P x(t)[6]$.

Proposition 2.1 The equilibrium $x(t)=0$ of the state feedback T-Sfuzzy system [6] is globally asymptotically stable if there exists a common symmetric positive definite matrix $P$ of appropriate dimensions satisfying

$$
\begin{aligned}
& H_{i k}^{T} P+P H_{i k}<0 ; \quad \forall i=1,2, \ldots, r ; k=1,2, \ldots, v \\
& G_{i j k}^{T} P+P G_{i j k}<0 ; \quad \forall i<j ; i, j=1, \ldots, r ; k=1, \ldots, v
\end{aligned}
$$

with $H_{i k}$ and $G_{i j k}$ for $i=1,2, \ldots, r$ and $k=1,2, \ldots, v$ as already defined.

Proof. Consider a Lyapunov function candidate of the type

$$
V(x(t))=x(t)^{T} P x(t)
$$


along the trajectories of system (6). Taking its derivative, we have

$$
\begin{aligned}
\dot{V}(x(t))= & \dot{x}(t)^{T} P x(t)+x(t)^{T} P \dot{x}(t) \\
= & x(t)^{T}\left[\sum_{i=1}^{r} \alpha_{1}^{2}(x(t))\left(\sum_{k=1}^{v} \eta_{k}(p) H_{i k}\right)^{T} P+P\left(\sum_{k=1}^{v} \eta_{k}(p) H_{i k}\right)\right. \\
& +\sum_{i<j}^{r} \alpha_{i}(x(t)) \alpha_{j}(x(t))\left(\sum_{k=1}^{v} \eta_{k}(p) G_{i j k}\right)^{T} P \\
& \left.+P\left(\sum_{k=1}^{v} \eta_{k}(p) G_{i j k}\right)\right] x(t) .
\end{aligned}
$$

Since (10) is a quadratic function, system (6) is asymptotically stable if we have

$$
\begin{gathered}
\sum_{i=1}^{r} \alpha_{i}^{2}(x(t))\left(\sum_{k=1}^{v} \eta_{k}(p) H_{i k}\right)^{T} P+P\left(\sum_{k=1}^{v} \eta_{k}(p) H_{i k}\right) \\
+\sum_{i<j}^{r} \alpha_{i}(x(t)) \alpha_{j}(x(t))\left(\sum_{k=1}^{\nu} \eta_{k}(p) G_{i j k}\right)^{T} P \\
+P\left(\sum_{k=1}^{v} \eta_{k}(p) G_{i j k}\right)<0
\end{gathered}
$$

Using the fact that $\alpha_{i}(x(t)) \geq 0$ and $\eta_{k}(p) \geq 0$ for all $i=1,2, \ldots, r$ and $k=1,2, \ldots, v$, we have

$$
H_{i k}^{T} P+P H_{i k}<0 ; \forall i=1,2, \ldots, r ; k=1,2, \ldots, v
$$

and

$$
G_{i j k}^{T} P+P G_{i j k}<0 ; \forall i<j ; i, j=1, \ldots, ; k=1, \ldots, v
$$

which completes the proof.

\subsection{Guaranteed cost fuzzy control design via LMIs}

In this section, we propose a robust fuzzy control design in terms of an optimal quadratic guaranteed cost problem as in Costa and Oliveira [11] and Jadbabaie et al. [12]. This approach is based on the global stability of the feedback T-S fuzzy system (6). In order to obtain a systematic control design, we formulate the problem in the context of the convex analysis using LMIs. 
Definition 2.1. The T-S fuzzy system with uncertainties (3) is said to be stable if there exists a stabilizing control law as in (4) such that an upper bound on the quadratic performance index

$$
C\left(x_{0}, u(t)\right)=\int_{0}^{\infty}\left(x(t)^{T} Q_{0} x(t)+u(t)^{T} R_{0} u(t)\right) d t
$$

along the system trajectories is minimized with $Q_{0} \in R^{n \times n}, R_{0} \in R^{m \times m}, Q_{0} \geq$ 0 and $R_{0}>0$ weighting symmetric matrices which are chosen to yield desired performances.

Definition 2.2. If there exist a stabilizing fuzzy control law (4) and a positive scalar $\delta$ such that $C\left(x_{0}, u(t)\right)<\delta$ for the T-S fuzzy system with uncertainties (3) then $\delta$ is a guaranteed cost and (4) is a guaranteed cost control.

Proposition 2.2. Consider the T-S fuzzy system with uncertainties (3), fuzzy control law (4) and cost performance (12). If there exist a symmetric positive definite matrix $W$ and matrices $X_{i}, i=1,2, \ldots, r$ of appropriate dimensions which satisfy

$$
\begin{gathered}
Q_{0} \geq 0 ; R_{0}>0 ; W>0 \\
{\left[\begin{array}{ccccc}
\theta_{i k} & W Q_{0}^{1 / 2} & X_{1}^{T} R_{0}^{1 / 2} & \ldots & X_{r}^{T} R_{0}^{1 / 2} \\
Q_{0}^{1 / 2} W & -I_{n} & 0 & 0 & \\
R_{0}^{1 / 2} X_{1} & 0 & -I_{m} & \ddots & 0 \\
\vdots & \vdots & \vdots & & \vdots \\
R_{0}^{1 / 2} X_{r} & 0 & 0 & \ldots & -I_{m}
\end{array}\right]<0 ; \forall i=1, \ldots, r ; k=1, \ldots, v} \\
\\
{\left[\begin{array}{ccccc}
\phi_{i j k} & W Q_{0}^{1 / 2} & X_{1}^{T} R_{0}^{1 / 2} & \ldots & X_{r}^{T} R_{0}^{1 / 2} \\
Q_{0}^{1 / 2} W & -I_{n} & 0 & & 0 \\
R_{0}^{1 / 2} X_{1} & 0 & -I_{m} & \ddots & 0 \\
\vdots & \vdots & \vdots & & \vdots \\
R_{0}^{1 / 2} X_{r} & 0 & 0 & \ldots & -I_{m}
\end{array}\right]<0 ; \forall i<j ; i, j=1, \ldots, r ; k=1, \ldots, v}
\end{gathered}
$$




$$
\begin{aligned}
\phi_{i j k}:= & W A_{i k}^{T}+A_{i k} W-X_{j}^{T} B_{i k}^{T}-B_{i k} X_{j} \\
& +W A_{i k}^{T}+A_{j k} W-X_{i}^{T} B_{j k}^{T}-B_{j k} X_{i}
\end{aligned}
$$

where $W:=P^{-1}$ and $X_{i}:=F_{i} W$, then the fuzzy control law (4) with $F_{i}=$ $X_{i} W^{-1}$ is a guaranteed control law and the cost given by $\delta:=x_{0}^{T} W^{-1} x_{0}$ is a guaranteed cost.

Proof. Assume that there exist a symmetric positive definite matrix $P=W^{-1}$ and matrices $F_{i}=X_{i} W^{-1}$ satisfying LMIs (13)-(15). Applying the Schur complement [13] in (14) and (15) and pre and post multiplying the resulting inequalities by $P$, after performing some algebraic manipulations, they can be reduced to

$$
\begin{gathered}
H_{i k}^{T} P+P H_{i k}+Q_{0}+\sum_{l=1}^{r} F_{l}^{T} R_{0} F_{l}<0 ; \\
i=1,2, \ldots, r ; k=1,2, \ldots, v
\end{gathered}
$$

and

$$
\begin{gathered}
G_{i j k}^{T} P+P G_{i j k}+Q_{0}+\sum_{l=1}^{r} F_{l}^{T} R_{0} F_{l}<0 \\
i<j ; i, j=1,2, \ldots, r ; k=1,2, \ldots, v .
\end{gathered}
$$

Multiplying (16) by $\alpha_{i}^{2}(x(t)) \eta_{k}(p)$ and (17) by $\alpha_{i}(x(t)) \alpha_{j}(x(t)) \eta_{k}(p)$ and using the fact that

$$
\sum_{k=1}^{v} \eta_{k}(p)=1 \quad \text { and } \quad \sum_{i=1}^{r} \alpha_{i}(x)=1,
$$

we have

$$
\begin{aligned}
\sum_{i=1}^{r} \alpha_{i}^{2}(x(t))\left(\sum_{k=1}^{\nu} \eta_{k}(p) H_{i k}\right)^{T} P+P\left(\sum_{k=1}^{v} \eta_{k}(p) H_{i k}\right) \\
+\sum_{i<j}^{r} \alpha_{i}(x(t)) \alpha_{j}(x(t))\left(\sum_{k=1}^{v} \eta_{k}(p) G_{i j k}\right)^{T} P \\
+P\left(\sum_{k=1}^{v} \eta_{k}(p) G_{i j k}\right)<-\left(Q_{0}+\sum_{l=1}^{r} F_{l}^{T} R_{0} F_{l}\right) .
\end{aligned}
$$


As $Q_{0}+\sum_{i=1}^{r} F_{i}^{T} R_{0} F>0$, by Proposition 2.1, the state feedback T-S fuzzy system (6) is asymptotically stable. Using Proposition 2.1, (18) can be written as

$$
x(t)^{T}\left(Q_{0}+\sum_{l=1}^{r} F_{l}^{T} R_{0} F_{l}\right) x(t)<-\dot{V}(x(t)) .
$$

Substituting (4) in (12), it results

$$
\begin{gathered}
C\left(x_{0}, u(t)\right)=\int_{0}^{\infty} x(t)^{T} \\
{\left[Q_{0}+\left(\sum_{l=1}^{r} \alpha_{l}(x(t)) F_{l}\right)^{T} R_{0}\left(\sum_{l=1}^{r} \alpha_{l}(x(t)) F_{l}\right)\right] x(t) d t}
\end{gathered}
$$

Using the fact that

$$
\sum_{l=1}^{r} F_{l}^{T} R_{0} F_{l}>\left(\sum_{l=1}^{r} \alpha_{l}(x(t)) F_{l}\right)^{T} R_{0}\left(\sum_{l=1}^{r} \alpha_{l}(x(t)) F_{l}\right)
$$

[12], we can write (19) as

$$
C\left(x_{0}, u(t)\right)=\int_{0}^{\infty} x(t)^{T}\left(Q_{0}+\sum_{l=1}^{r} F_{l}^{T} R_{0} F_{l}\right) x(t) d t .
$$

Thus, using (22) and integrating both sides of (19), we have

$$
C\left(x_{0}, u(t)\right)<x_{0}^{T} P x_{0}
$$

The result follows by Definitions 2.1 and 2.2.

Lemma 2.1. If $\delta=x_{0}^{T} W^{-1} x_{0}$ is a guaranteed cost for the feedback T-S fuzzy system (6) under performance index (9) then

$$
\delta_{\max }=\lambda_{\max }\left(W^{-1}\right)\left\|x_{0}\right\|^{2}
$$

is a guaranteed cost for (6) and an upper bound for $\delta$ with $\lambda_{\max }($.$) the maximum$ eigenvalue. 
Proof. Using singular value decompositions the result follows straightforward.

Using both Proposition 2.2 and Lemma 2.1, we can construct the following generalized eigenvalue problem (GEVP) [13] in order to obtain the GCFC for the state feedback T-S fuzzy system with uncertainties (6)

$$
\min _{W, X_{i}} \gamma \text { subject to } I \leq \gamma W, \quad(13)-(15)
$$

If (25) is feasible, we have $\gamma>\lambda_{\max }\left(W^{-1}\right)$ and the feedback gains $F_{i}=X_{i} W^{-1}$, for $i=1,2, \ldots, r$.

\section{Simulation results}

In this section, an illustrative example of the applicability of the proposed approach is given. We consider the same example as in Tanaka et al. [8], a simple nonlinear mass-spring-damper mechanical system with an uncertain parameter shown in Figure 1, which is described as

$$
M \ddot{y}(t)+g(y(t), \dot{y}(t))+f(y(t))=\phi(\dot{y}(t)) u(t)
$$

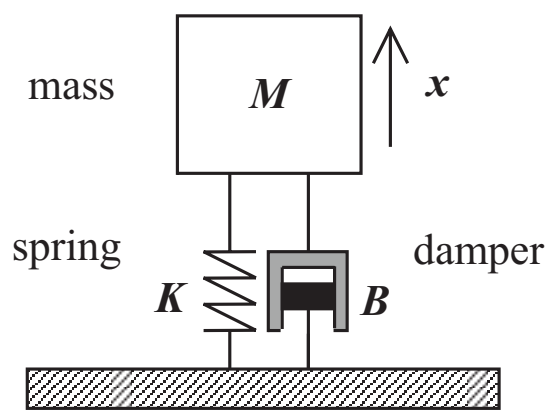

Figure 1 - Mass-spring-damper system.

where $M$ is the mass, $u$ is the force, $f($.$) is the nonlinear or uncertain term with$ respect to the spring, $g($.) is the nonlinear or uncertain term with respect to the damper and $\phi($.$) is the nonlinear term with respect to the input system. We$ assume $f(y(t))=c(t) y(t)$ with

$$
c(t) \in[0.51 .81], g(y(t), \dot{y}(t))=\dot{y}(t), \phi(\dot{y}(t))=1+0.13 \dot{y}^{3}(t)
$$


and $M=1$, for $y(t) \in[-1.51 .5]$ and $\dot{y}(t) \in[-1.51 .5]$ as in Tanaka et al. [8]. Defining $x(t):=[\dot{y}(t) y(t)]^{T}$, we can write (26) in the following state space representation

$$
\left[\begin{array}{l}
\dot{x}_{1}(t) \\
\dot{x}_{2}(t)
\end{array}\right]=\left[\begin{array}{cc}
-1 & -c(t) \\
1 & 0
\end{array}\right]\left[\begin{array}{l}
x_{1}(t) \\
x_{2}(t)
\end{array}\right]+\left[\begin{array}{c}
1+0.13 x_{1}^{3}(t) \\
0
\end{array}\right] u(t) .
$$

As system (27) presents one uncertain parameter, we have two vertexes in the polytopic description as presented in Section 1. The local uncertain linear models were constructed by selecting $\bar{x}=\left[\begin{array}{lll}1.9740 & 0\end{array}\right]^{T}$ and $\bar{x}=[-1.97400]^{T}$ as operating points for $i=1,2$ and $k=1,2$

$$
\begin{aligned}
& A_{11}=\left[\begin{array}{cc}
-1 & -0.5 \\
1 & 0
\end{array}\right] ; B_{11}=\left[\begin{array}{c}
1.4387 \\
0
\end{array}\right] \\
& A_{12}=\left[\begin{array}{cc}
-1 & -1.81 \\
1 & 0
\end{array}\right] ; B_{12}=B_{11} \\
& A_{21}=A_{11} ; B_{21}=\left[\begin{array}{c}
0.5613 \\
0
\end{array}\right] \\
& A_{22}=A_{12} ; B_{22}=B_{21} .
\end{aligned}
$$

The performance of the global T-S fuzzy system is verified adopting

$$
\begin{aligned}
\alpha_{1}(x(t)) & =0.5+x_{1}(t)^{3} / 6.75 \\
\alpha_{2}(x(t)) & =0.5-x_{1}(t)^{3} / 6.75 \\
c(t) & =1.155+0.655 \cos \left(3 x_{2}(t)^{10 \operatorname{sen}\left(x_{1}(t)\right)}\right)
\end{aligned}
$$

for $x_{1} \in[-1.51 .5]$ [8].

As shown in Section 2, the design control procedure is systematically accomplished by using LMIs to solve the optimization problem (25). We choose

$$
Q_{0}=I_{2}, R_{0}=0.07 \quad \text { and } \quad x_{0}=[-0.5-1.0]^{T}
$$

for both rules. Using the Matlab ${ }^{\circledR}$ LMI toolbox as well as the ordinary differential equation (ODE) solver, we obtain the main results summarized in Table 1. Figures 2 and 3 show the feedback uncertain nonlinear system responses. The 
proposed solution is comparable to the one given in Tanaka et al. [8]. Its advantage is that it follows a systematic procedure and it minimizes a quadratic performance objective or cost function (9).

\begin{tabular}{|c|}
\hline Feedback gains \\
\hline$F_{1}=\left[\begin{array}{ll}1.6042 & 1.0833\end{array}\right]$ \\
$F_{2}=\left[\begin{array}{ll}4.1000 & 2.7711\end{array}\right]$ \\
\hline Solution if the optimization problem \\
\hline$P=\left[\begin{array}{ll}0.5892 & 0.3982 \\
0.3982 & 1.4966\end{array}\right]$ \\
\hline Obtained guaranted costs \\
\hline$\delta=1.7885$ \\
$\delta_{\max }=1.7947$ \\
\hline
\end{tabular}

Table 1 - Design and stability analysis: main results.
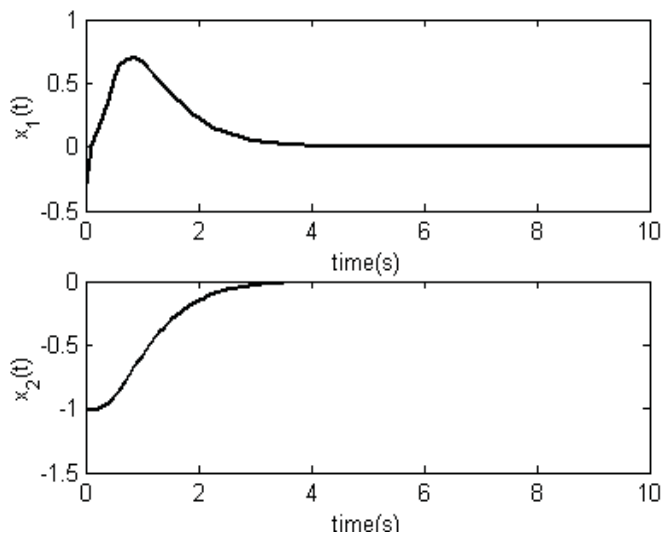

Figure 2 - Mass-spring-damper space states.

\section{Conclusion}

This paper addresses the design of guaranteed cost fuzzy controllers for a class of uncertain nonlinear systems using LMIs. We establish a sufficient condition for the stability of the global T-S fuzzy system using design methods available for linear uncertain systems. The GCFC problem is formulated in terms of the minimization of an upper bound on the guaranteed cost. The proposed 

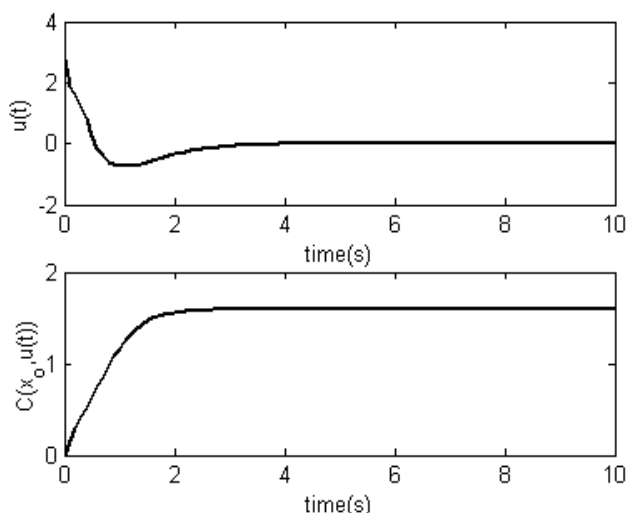

Figure 3 - Force applied to the mass-spring-dumper system and performance index.

approach yields a computationally tractable solution to the control problem for uncertain nonlinear systems using linguistic rules for the nonlinearities and a polytopic description for the uncertainties. Different control techniques can also be explored using the framework presented.

\section{Acknowledgments}

This work was supported by the Fundação do Amparo à Pesquisa do Estado de São Paulo under grant 00/05060-1.

\section{REFERENCES}

[1] T. Takagi and M. Sugeno, Fuzzy identification of systems and its application to modeling and control. IEEE Trans. Systems, Man and Cybernetics, 15 (1) (1985), 116-132.

[2] G. Feng, S.G. Cao, N.W. Rees and C.K. Chak, Design of fuzzy control systems with guaranteed stability. Fuzzy Sets and Systems, 85 (1) (1997), 1-10.

[3] N.W. Rees, S.G. Cao and G. Feng, Further results about quadratic stability of continuous-time fuzzy control systems. Int. J. Systems Science, 28 (4) (1997), 397-404.

[4] S. Hong and R. Langari, An LMI-based $\mathrm{H}_{\infty}$ fuzzy control system design with TS framework. Information Sciences, 123 (3-4) (2000), 163-179.

[5] J. Li and H.O. Wang, D. Niemann and K. Tanaka, Dynamic parallel distributed compensation for Takagi-Sugeno fuzzy systems: an LMI approach. Information Sciences, 123 (3-4) (2000), 201-221. 
[6] K. Tanaka, H.O. Wang and M.F. Griffin, An approach to fuzzy control of nonlinear: stability and design issues. IEEE Trans. Fuzzy Systems, 4 (1) (1996), 14-23.

[7] K.R. Lee, E.T. Jeung and H.B. Park, Robust fuzzy $H_{\infty}$ control for uncertain nonlinear systems via state feedbak: an LMI approach. Fuzzy Sets and Systems, 120 (1) (2001), 123-134.

[8] T. Ikeda, K. Tanaka and H.O. Wang, Robust stabilization of a class of uncertain nonlinear systems via fuzzy control: Quadratic stabilizability, $H_{\infty}$ control theory, and linear matrix inequalities, IEEE Trans. Fuzzy Systems, 4 (1) (1996), 1-13.

[9] N.W. Rees, S.G. Cao and G. Feng, $H_{\infty}$ control of uncertain fuzzy continuous-time systems. Fuzzy Sets and Systems, 115 (2) (2000), 171-190.

[10] M.C.M. Teixeira and S.H. Zak, Stabilizing controller design for uncertain nonlinear systems using fuzzy models. IEEE Trans. Fuzzy Systems, 7 (2) (1999), 133-142.

[11] E.F. Costa and V.A. Oliveira, On the design of guaranteed cost controllers for a class of uncertain linear systems. Systems and Control Letters, 46 (1) (2002), 17-29.

[12] M. Jamshid, A. Jadbabaie and A. Titli, Guaranteed-cost design of continuous-time Takagi Sugeno fruzzy controllers via linear matrix inequalities. Proc. IEEE Int. Conf. Fuzzy Systems, (1998), 268-273.

[13] E. Feron, S. Boyd, L.E. Ghaoui and V. Balakrishnan, Linear matrix inequalities in system and control theory. SIAM, Philadelphia, PA, (1994) 